\title{
Article \\ Plasma Technology Increases the Efficacy of Prothioconazole against Fusarium graminearum and Fusarium proliferatum Contamination of Maize (Zea mays) Seedlings
}

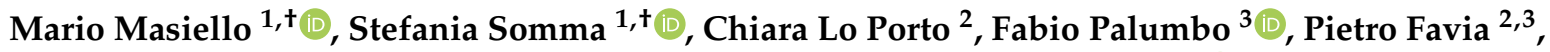 \\ Francesco Fracassi ${ }^{2,3}$, Antonio Francesco Logrieco ${ }^{1}$ and Antonio Moretti ${ }^{1, * \mathbb{D}}$ \\ 1 Institute of Sciences of Food Production (ISPA), National Research Council of Italy, Via Amendola 122/O, \\ 70126 Bari, Italy; mario.masiello@ispa.cnr.it (M.M.); stefania.somma@ispa.cnr.it (S.S.); \\ antonio.logrieco@ispa.cnr.it (A.F.L.) \\ 2 Department of Chemistry, University of Bari Aldo Moro, Via Orabona 4, 70126 Bari, Italy; \\ c.loporto@ba.ipcf.cnr.it (C.L.P.); pietro.favia@uniba.it (P.F.); francesco.fracassi@uniba.it (F.F.) \\ 3 Institute of Nanotechnology, National Research Council of Italy, c/o Department of Chemistry, University of \\ Bari Aldo Moro, Via Orabona 4, 70126 Bari, Italy; fabio.palumbo@cnr.it \\ * Correspondence: antonio.moretti@ispa.cnr.it; Tel.: +39-3386701273 \\ + These authors contributed equally to this work.
}

Citation: Masiello, M.; Somma, S.; Lo Porto, C.; Palumbo, F.; Favia, P.; Fracassi, F.; Logrieco, A.F.; Moretti, A. Plasma Technology Increases the Efficacy of Prothioconazole against Fusarium graminearum and Fusarium proliferatum Contamination of Maize (Zea mays) Seedlings. Int. J. Mol. Sci. 2021, 22, 9301. https://doi.org/ $10.3390 /$ ijms 22179301

Academic Editors: Ricardo Molina and Ryszard Lobinski

Received: 9 June 2021

Accepted: 25 August 2021

Published: 27 August 2021

Publisher's Note: MDPI stays neutral with regard to jurisdictional claims in published maps and institutional affiliations.

Copyright: (c) 2021 by the authors. Licensee MDPI, Basel, Switzerland This article is an open access article distributed under the terms and conditions of the Creative Commons Attribution (CC BY) license (https:// creativecommons.org/licenses/by/ $4.0 /)$
Abstract: The contamination of maize by Fusarium species able to produce mycotoxins raises great concern worldwide since they can accumulate these toxic metabolites in field crop products. Furthermore, little information exists today on the ability of Fusarium proliferatum and Fusarium graminearum, two well know mycotoxigenic species, to translocate from the seeds to the plants up to the kernels. Marketing seeds coated with fungicide molecules is a common practice; however, since there is a growing need for reducing chemicals in agriculture, new eco-friendly strategies are increasingly tested. Technologies based on ionized gases, known as plasmas, have been used for decades, with newer material surfaces, products, and approaches developed continuously. In this research, we tested a plasma-generated bilayer coating for encapsulating prothioconazole at the surface of maize seeds, to protect them from F. graminearum and F. proliferatum infection. A minimum amount of chemical was used, in direct contact with the seeds, with no dispersion in the soil. The ability of F. graminearum and F. proliferatum species to translocate from seeds to seedlings of maize has been clearly proven in our in vitro experiments. As for the use of plasma technology, the combined use of the plasma-generated coating with embedded prothioconazole was the most efficient approach, with a higher reduction of the infection of the maize seminal root system and stems. The debated capability of the two Fusarium species to translocate from seeds to seedlings has been demonstrated. The plasma-generated coating with embedded prothioconazole resulted in a promising sustainable approach for the protection of maize seedlings.

Keywords: eco-friendly strategies; germinability; mycotoxins; systemic transmission

\section{Introduction}

Maize is one of the most cultivated crops worldwide, and a staple food for human and livestock in several geographical areas. In addition, environmentally sustainable policies have led to the proposition of new maize by-products, such as starch, fibers, and oil in the food and chemical industry [1,2].

Maize can be colonized by several fungal pathogens, among which species belonging to the Fusarium genus are of particular concern. Many of them, particularly the soil and seed-borne pathogens, are associated with some of the most devastating fungal diseases of maize, such as Fusarium ear and stalk rots, Fusarium stem and root rots, and seedling decay, and cause significant losses of products all over the world. In addition, many Fusarium species produce a wide range of mycotoxins-harmful secondary metabolites that 
accumulate in plants throughout their growth. The seedling stage, flowering time, and wax ripe stage of kernels are all highly susceptible periods during which maize plants can be colonized by Fusarium species [3]. For this reason, maize intended for food and feed results in frequently being contaminated by Fusarium mycotoxins, as well as the maize-based by-products, since these mycotoxins often remain stable also at the high temperature used in food processing [4]. Fumonisins (FBs), deoxynivalenol (DON), and zearalenone (ZEA) are the most important mycotoxins accumulated by Fusarium species in both stalks and kernels, at harvest, and in the field [4].

While FBs are mainly produced by F. verticillioides and F. proliferatum, F. graminearum is the main species responsible for the production of DON and ZEA [4]. These species can infect maize plants through the root system from the soil, from contaminated seeds, or through spores carried over by wind, water, and insect wounds. The ability of F. verticillioides to systemically colonize seedlings from seeds is well known and has been deeply investigated by several authors for decades [5-7]. On the other hand, the knowledge of the transmission path of F. graminearum and F. proliferatum from seeds to seedlings is poor and controversial, although some papers report that the latter species shares a similar disease cycle with F. verticillioides [8]. Indeed, F. proliferatum and F. verticillioides have been often reported as associated in colonizing maize plants worldwide [8-12]. F. proliferatum is a ubiquitous species, able to colonize a wide range of important agricultural crops besides maize and other cereals, such as vegetables and fruit trees [13]. Moreover, F. proliferatum produces also other mycotoxins, such as moniliformin [14], beauvericin [15], fusaproliferin [16], and fusarins [17], thus increasing the risk of multiple mycotoxin occurrence in foodstuff. Besides the abovementioned species, F. graminearum has been reported as a maize pathogen in geographical areas where the environmental conditions are colder or when the maize is cultivated as a second crop and is exposed to low temperatures and high humidity [18]. According to the single species, Fusarium infections occurring in the first growth stages of maize can contribute to the final mycotoxin contamination in kernels, since this early colonization of plant tissues is usually followed by the further progress of the infection up to the ears at harvest, in certain environmental conditions [19]. Therefore, coating the seeds with fungicides and/or other molecules, such as bio-stimulants, is a common agronomic practice for all marketed maize seeds, to improve both their germinability and the vigor of seedlings.

Few chemical compounds are registered to control fungal diseases on maize and other cereals; moreover, at the same time, the occurrence of resistance to fungicides in fungal populations can reduce the effectiveness of the molecules widely used for seed treatments. Both integrated pest management guidelines and environmental sustainability policies encourage researchers to develop newer eco-friendly strategies to control both fungal species and their mycotoxin accumulation, in order to reduce chemical treatments and, consequently, their negative environmental impact.

The word plasma refers to ionized gases in thermodynamic equilibrium conditions (thermal) or far from them (cold). Technologies based on cold plasmas, in particular, impact our everyday life with so many surface-modification processes and commercial products in strategic areas such as microelectronics, semiconductors, polymers, and biomaterials, just to mention a few [20]. Plasma deposition, treatment, and etching processes are utilized for altering the surface properties of materials with an added or ablated thin layer, usually thinner than one micron, in the range of tens to hundreds of nanometers. Since surfacemodification plasma techniques use a minimum amount of reagents and no solvents, they are considered eco-friendly. Due to their low thermal impact, also, they allow the modification of the surface of thermo-labile substrates with no changes to their bulk properties. In the last two decades, cold plasma technologies have impacted also life sciences, such as medicine, agriculture, and food science, with newer approaches and processes in anti-cancer therapies, as well as in the decontamination of wounds, field water, seeds, and fresh food [20-22]. 
Cold plasma technologies are being widely tested on seeds to enhance the germination rate and plant growth [23-27]. They have been also applied for inactivating pathogenic and spoilage microorganisms in food [28-30]. All proposed applications of cold plasma technology for agriculture, including water treatments, are presented in Puac et al. [31].

We carried out a preliminary study applying a fungicide on maize seeds using plasma technology, by embedding it with a coating plasma-deposited directly on the seeds, for better investigation of the potentiality of this technology in controlling fungal infections and reducing the use of fungicides in agriculture [32]. Among marketed fungicides, Masiello et al. reported that the de-methylation inhibitor prothioconazole was the most active molecule in vitro against the most important mycotoxigenic fungi of maize and confirmed its effectiveness in reducing F. graminearum and F. proliferatum contamination of maize plants in the field [33]. Based on this knowledge, Lo Porto et al. [32] developed a plasma-deposition approach to apply prothioconazole directly and only at the surface of the maize seeds, to enhance the effectiveness of the fungicide, to limit its used quantity, and to avoid its dispersion in the soil. Indeed, this approach is original so far in the literature, since plasma treatments of seeds, generally in the form of ablation and hydrophilization of their surfaces, are generally tested for direct decontamination or for inducing faster germination, but not plasma deposition. Most surface modification (etching, grafting, and deposition) cold plasma processes have been developed at low (10-1000 mTorr) pressure. In the last decades, however, for several reasons, processes at atmospheric pressure have been developed too $[34,35]$. Two low pressure plasma-deposition processes were used by Lo Porto et al. [32] to develop first a thin wettable layer around the seeds, where the prothioconazole could be sprayed and remain, and then a hydrophobic layer aimed to embed the fungicide, and repristinate the natural hydrophobicity of the seed surface. The effective total thickness of the two layers is in the order of hundreds of nanometers. This approach allowed the effective plasma-assisted immobilization of the fungicide on the maize seeds without interference with their water absorption and germination, and the reduction of the effective dose of the fungicide. In order to test the full applicability of such an approach to control infection from external sources of both F. graminearum and F. proliferatum, and determine if they could be systemically transmitted, we carried out experiments both in vitro and in planta with the following aims:

To evaluate the possible increased effectiveness of prothioconazole embedded in close contact with the seeds, with a plasma-deposition protocol, against $F$. graminearum and $F$. proliferatum;

To test the effects of the plasma-deposition protocol on seed germination and the transmission of $F$. graminearum and $F$. proliferatum infection from maize seeds;

To evaluate the possible systemic transmission of F. graminearum and F. proliferatum from seeds to seedlings.

\section{Results}

2.1. In Vitro Effect of Plasma Coating and Prothioconazole Application, Alone or in Combination, against Fusarium Species

Fusarium proliferatum was detected in $7 \%$ of seeds in the control (Thesis 1 ), while $F$. graminearum was not found. Moreover, an unexpected heavy contamination by Rhizopus occurred in Theses 1, 3A, and 3B, with contamination values of $100 \%, 13 \%$, and $20 \%$, respectively (Figure 1 ).

After 3 days of incubation, Fusarium strains had colonized the entire surface of PDA plates. For both Fusarium species, the treatment with no plasma coating and no prothioconazole (Theses $2 \mathrm{~A}$ and $2 \mathrm{~B}$ ), and the treatment with only plasma coating and no fungicide (Theses $4 \mathrm{~A}$ and $4 \mathrm{~B}$ ) were completely $(100 \%)$ colonized by Fusarium; after 10 days of incubation, the seeds were completely colonized by Fusarium mycelium. In the theses treated with prothioconazole only, the fungicide showed a good efficacy to reduce fungal colonization (Theses $3 \mathrm{~A}$ and $3 \mathrm{~B}$ ), and the seeds remained healthy and all germinated. In particular, around each seed, an inhibition halo of about $2.5 \mathrm{~cm}$ of diameter was observed after 10 days of incubation. Indeed, the best activity to control both Fusarium species was obtained in 
Theses $5 \mathrm{~A}$ and $5 \mathrm{~B}$, where the seeds treated with the prothioconazole embedded in the plasma-deposited coating were grown. These seeds resulted in being completely free of Fusarium mycelium, and around each of the seeds an inhibition halo with a diameter of $3.5 \mathrm{~cm}$ was observed.
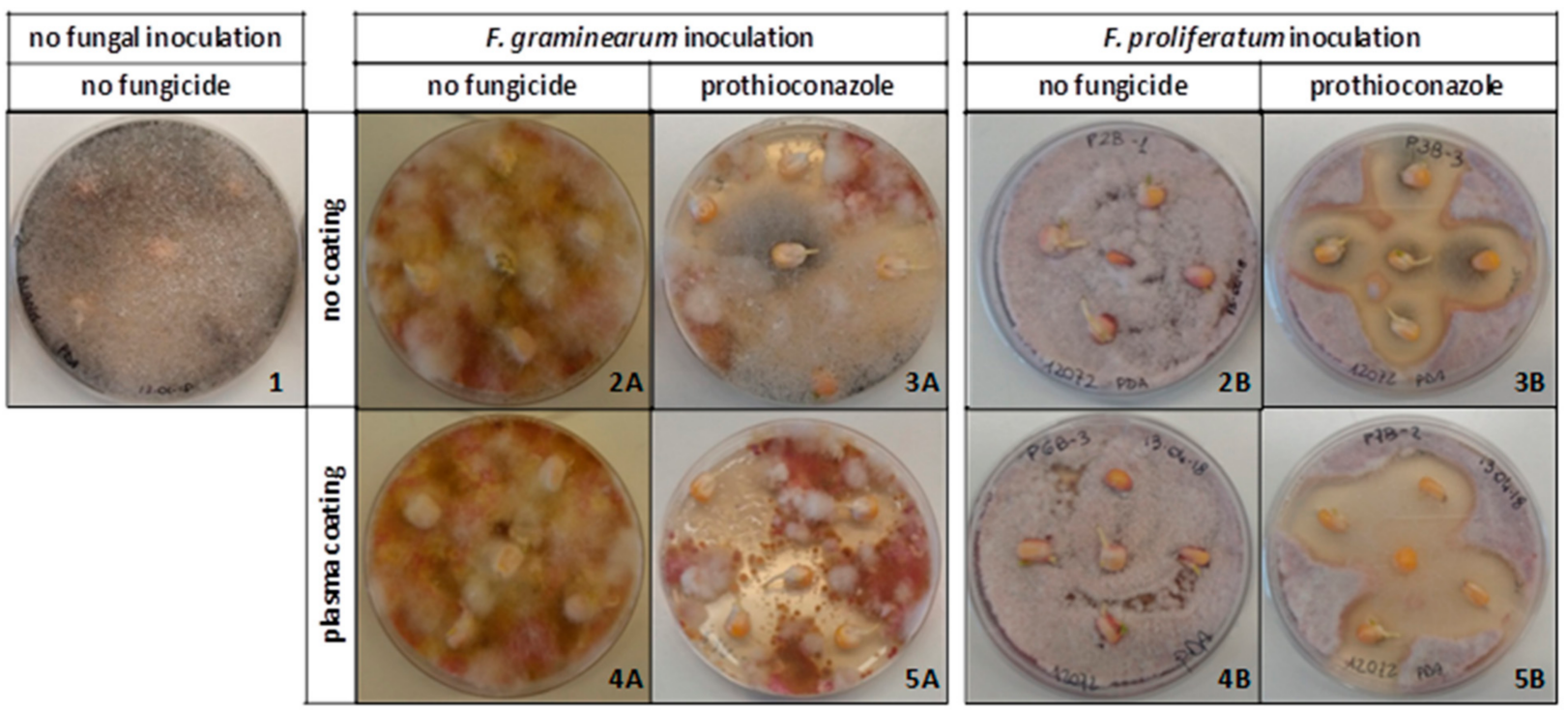

Figure 1. Maize seed contamination evaluated after 3 days, on different Theses, inoculated and not inoculated with Fusarium, coated and not by means of plasma deposition, treated and not with prothioconazole. The thesis number is shown at the bottom right of each picture.

Data on seed germination and fungal contamination assessed for each thesis in the two experiments for F. graminearum (A) and F. proliferatum (B) are reported in Figure 2.

\subsubsection{Seed Germination}

Seed germination rate was assessed 7 days after seed sowing. All seeds of the control (Thesis 1) germinated (100\%).

Fusarium graminearum. Seeds inoculated with F. graminearum (Figure 2A) showed $100 \%$ germination when prothioconazole was applied alone (Thesis $3 \mathrm{~A}$ ) or in combination with the plasma-deposited coating (Thesis $5 \mathrm{~A}$ ), and $93 \%$ for the seeds that were not treated with the fungicide (Thesis 2A), in presence of the plasma-deposited bilayer (Thesis 4A). We calculated the standard error (SE) values for all theses. Theses $2 \mathrm{~A}$ and $5 \mathrm{~A}$ had SE values of 6.7 .

Fusarium proliferatum. In the theses inoculated with the conidial suspension of $F$. proliferatum (Figure 2B), when prothioconazole was applied alone or embedded in the plasma-deposited bilayer, seed germination rates of $93 \%$ (Thesis 3B; SE 6.7) and 87\% (Thesis 5B; SE 6.7) were measured, respectively. The lowest germination values of $87 \%$ (Thesis 2B; SE 13.3) and 67\% (Thesis 4B; SE 6.7) were observed in the case of seeds untreated or treated only with the plasma coating.

\subsubsection{Transmission of the Fungi to Roots and Stems}

In this experiment, the capability of the two Fusarium species to colonize seedling tissues from the seeds was evaluated.

Control. In the control (Thesis 1), F. graminearum was not detected in any root and seedling tissues, while F. proliferatum was detected in $7 \%$ of both seminal root systems and stem tissues (Figure 2). 

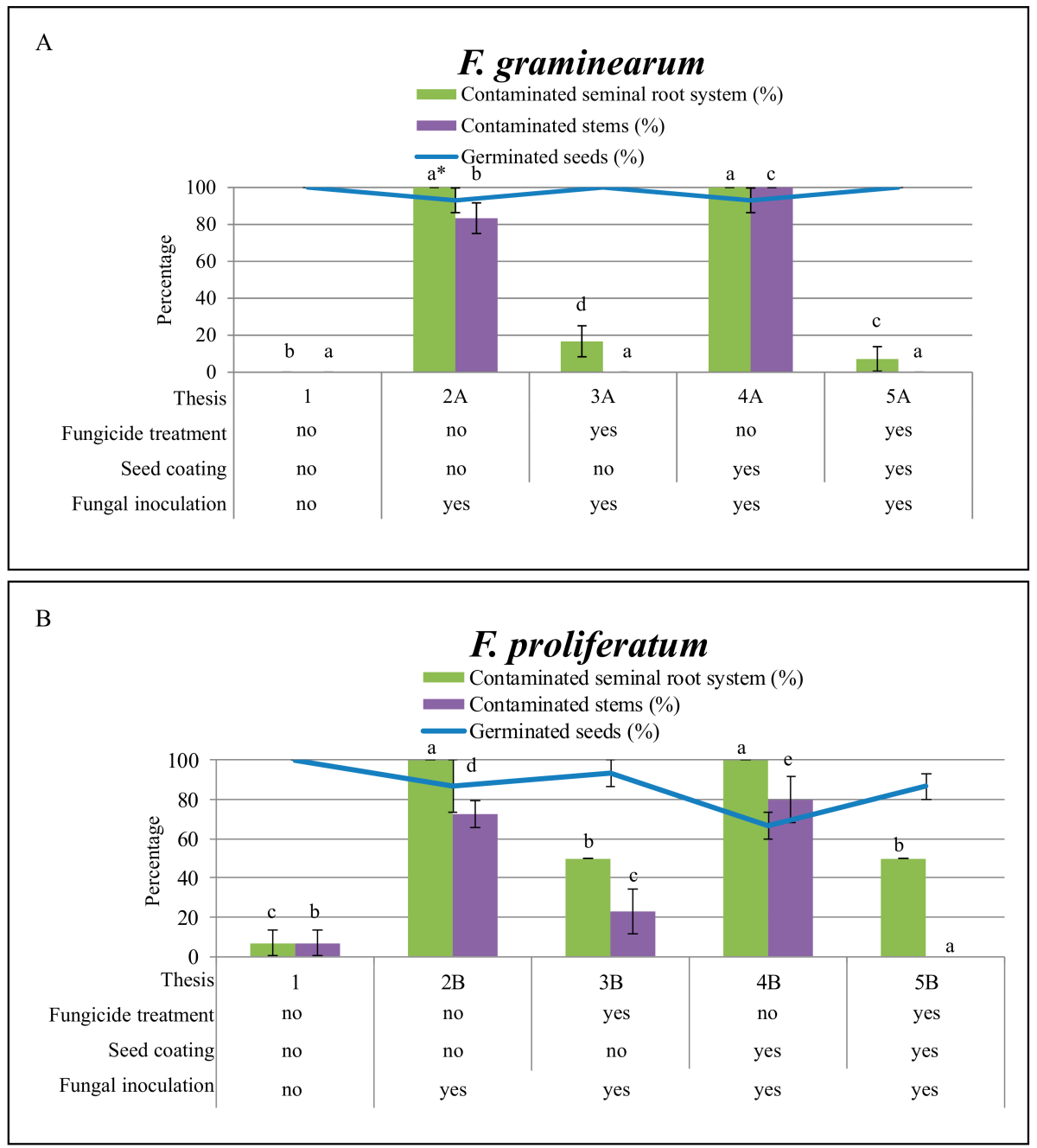

Figure 2. Percentage of seed germination and fungal contamination in maize seedlings grown in Petri dishes containing synthetic medium, after different and combined treatments (fungicide application, plasma coating, fungal inoculation). Two separated experiments were carried out for $F$. graminearum (A) and F. proliferatum (B). * Values with different letters are statistically different with $p<0.05$ as determined by one-way analysis of variance (ANOVA) followed by the Tukey test.

\section{Fusarium graminearum.}

Roots. For F. graminearum, the seminal root system of seedlings of both theses untreated with prothioconazole was found completely colonized, showing contamination values of $100 \%$. In the presence of prothioconazole instead, used alone or embedded in the bilayer coating, the colonization of the seminal root system by the pathogen had poor results (17 and $7 \%$, respectively). In particular, a statistically significant difference between Theses 3A (SE 8.3) and 5A (SE 6.7) was observed, in favor of the fungicide embedded in the coatings.

Stems. For F. graminearum, the stems of seedlings of both theses untreated with prothioconazole were highly colonized and showed contamination values of $100 \%$ (Thesis $4 \mathrm{~A}$ ) and $83 \%$ for the stems of Thesis 2A (SE 8.3). On the contrary, in theses treated with prothioconazole, used alone or embedded in the bilayer coating, there was not colonization of the stems by F. graminearum.

Fusarium proliferatum.

Roots. The seminal root systems of the seedlings with no prothioconazole treatment (Theses $2 \mathrm{~B}$ and $4 \mathrm{~B}$ ) were both totally colonized by F. proliferatum $(100 \%)$, with no statistical difference between each other. Instead, contamination values of $50 \%$ were detected on the 
seedling root systems for the seeds treated with prothioconazole (Theses $3 \mathrm{~B}$ and $5 \mathrm{~B}$, values of SE were 0 for both theses), combined or not with the plasma coating.

Stems. Fusarium proliferatum was found to be able to colonize the stems moving from the seeds to stem tissues of the seedlings. In the theses untreated with prothioconazole, stem contamination values of $73 \%$ were found in Thesis 2 B (SE 6.7) and of $80 \%$ in Thesis 4B (SE 11.5). Seedling stems of seeds exposed to prothioconazole alone, Thesis 3B, were found poorly colonized (23\%, SE 11.7); in addition, F. proliferatum was not detected in the stems of the seedlings resulting from seeds treated with prothioconazole embedded in the plasma bilayer (Thesis 5B).

\subsection{In Planta Effects of Plasma Coating and Prothioconazole Application}

The in-planta results are summarized in Figure 3. The efficacy of the plasma-based coating combined with the application of prothioconazole directly on the maize seeds was tested on maize seedlings grown in pots containing soil, to mimic field growth conditions.

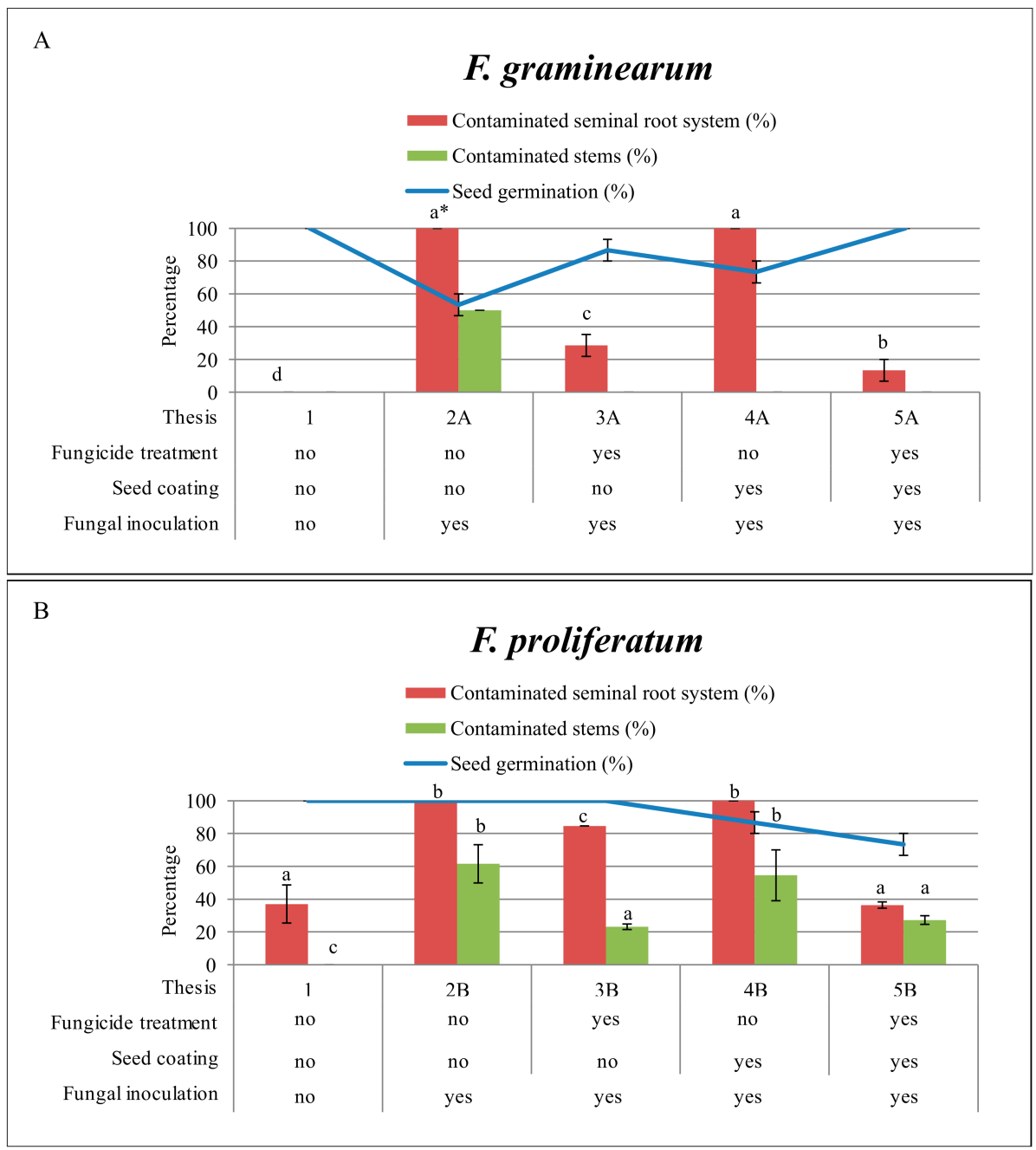

Figure 3. Percentage of seed germination and fungal contamination in maize seedlings grown in pots containing soil, after different and combined treatments (fungicide application, seed coating, fungal inoculation). Two separated experiments were carried out for F. graminearum (A) and F. proliferatum (B). ${ }^{*}$ Values with different letters are statistically different with $p<0.05$ as determined by one-way analysis of variance (ANOVA) followed by the Tukey test.

\subsubsection{Seed Germination}

Seed germination rate, shown in Figure 3, was measured 7 days after the emergence of the seedlings. In the control (Thesis 1), all seeds germinated (100\%). 
Fusarium graminearum. In the experiment with F. graminearum (Figure $3 \mathrm{~A}$ ), for seeds not treated with prothioconazole nor plasma (Thesis $2 \mathrm{~A}$ ) or treated only with the plasma coating (4A), the germination of seeds was found to be decreased, with values of $53 \%$ (SE 6.7) and $73 \%$ (SE 6.7), respectively. For seeds treated with prothioconazole, alone (Thesis $3 \mathrm{~A}$ ) or in combination with the plasma deposition (Thesis $5 \mathrm{~A}$ ), germination rates of $87 \%$ (SE 6.7) and 100\% were measured, respectively.

Fusarium proliferatum. In the experiment with F. proliferatum (Figure 3B), seed germination of $100 \%$ was measured for seeds untreated or treated with prothioconazole, without plasma coating (Thesis 2B and 3B). A decreased germination rate was observed in both theses where the seeds were plasma coated, alone or in combination with embedded prothioconazole, with germination values of $87 \%$ (Thesis $4 \mathrm{~B}$; SE 6.7) and 73\% (Thesis 5B; SE 6.7), respectively.

\subsubsection{Colonization of Roots and Stems}

Fusarium graminearum.

Roots. In the experiment with $F$. graminearum (Figure 3A), for seeds not treated with prothioconazole, $100 \%$ of the root system was found colonized by the fungi. Statistical differences were not observed between inoculated control theses and theses treated only with the plasma coating. For seeds treated with prothioconazole, alone or in combination with the plasma-deposited bilayer, statistically different root system contamination values of $29 \%$ (Thesis 3A; SE 6.7) and 13\% (Thesis 5A; SE 6.7) were found, respectively. Fusarium graminearum was absent in the root of seeds that were not inoculated.

Stems. With regard to stem colonization, fungal contamination was observed only in Thesis 2A, seeds with no prothioconazole nor plasma coating, but inoculated with F. graminearum, with a value of $50 \%$ (SE value was 0 ). Although inoculated with F. graminearum, Theses $3 \mathrm{~A}, 4 \mathrm{~A}$, and $5 \mathrm{~A}$ did not show any fungal contamination.

\section{Fusarium proliferatum.}

Roots. In the experiment with $F$. proliferatum (Figure 3B), F. proliferatum was detected also in the not inoculated Thesis 1, with the value of $37 \%$ in the root system (Thesis 1 ; SE 11.5). Theses $2 \mathrm{~B}$ and $4 \mathrm{~B}$, both not exposed to prothioconazole, revealed that $100 \%$ of the root system was contaminated. Theses $3 \mathrm{~B}$ and $5 \mathrm{~B}$, where prothioconazole was used, alone or in combination with the plasma coating, showed root system contamination values of $85 \%$ (Thesis 3B; SE 0) and 36\% (Thesis 5B; SE 1.9), respectively. Interestingly, Theses 1 (not inoculated seeds) and 5B, were statistically grouped in the same homogeneous group, demonstrating the strong effect of prothioconazole combined with the plasma coating on the seeds against Fusarium colonization.

Stems. With respect to stem contamination, $62 \%$ of stem contamination was recorded for untreated seeds (Thesis 2B; SE 11.7), while in seeds protected by the plasma coating (Thesis 4B), 55\% (SE 15.5) of stems were colonized by F. proliferatum. No statistical difference was observed between Theses $2 \mathrm{~A}$ and $4 \mathrm{~A}$. Theses $3 \mathrm{~B}$ and $5 \mathrm{~B}$, treated with prothioconazole, alone or in combination with the plasma bilayer, had values of 23\% (SE 1.7) and 27\% (SE 2.7) of stems colonized by F. proliferatum, respectively.

\subsubsection{Disease Symptoms on Seedlings}

The general appearance of the seedlings was evaluated 10 days after their emergence. The plants of the control (Thesis 1), as well as those inoculated with F. proliferatum (Theses $2 \mathrm{~B}, 3 \mathrm{~B}, 4 \mathrm{~B}, 5 \mathrm{~B})$, appeared vigorous, intensely green colored, 10-15 cm high, and with no evident symptoms of Fusarium infection. The plants inoculated with F. graminearum, instead, appeared pale green, $3-8 \mathrm{~cm}$ high, and with red mycelium present on the stems (Figure 4). 

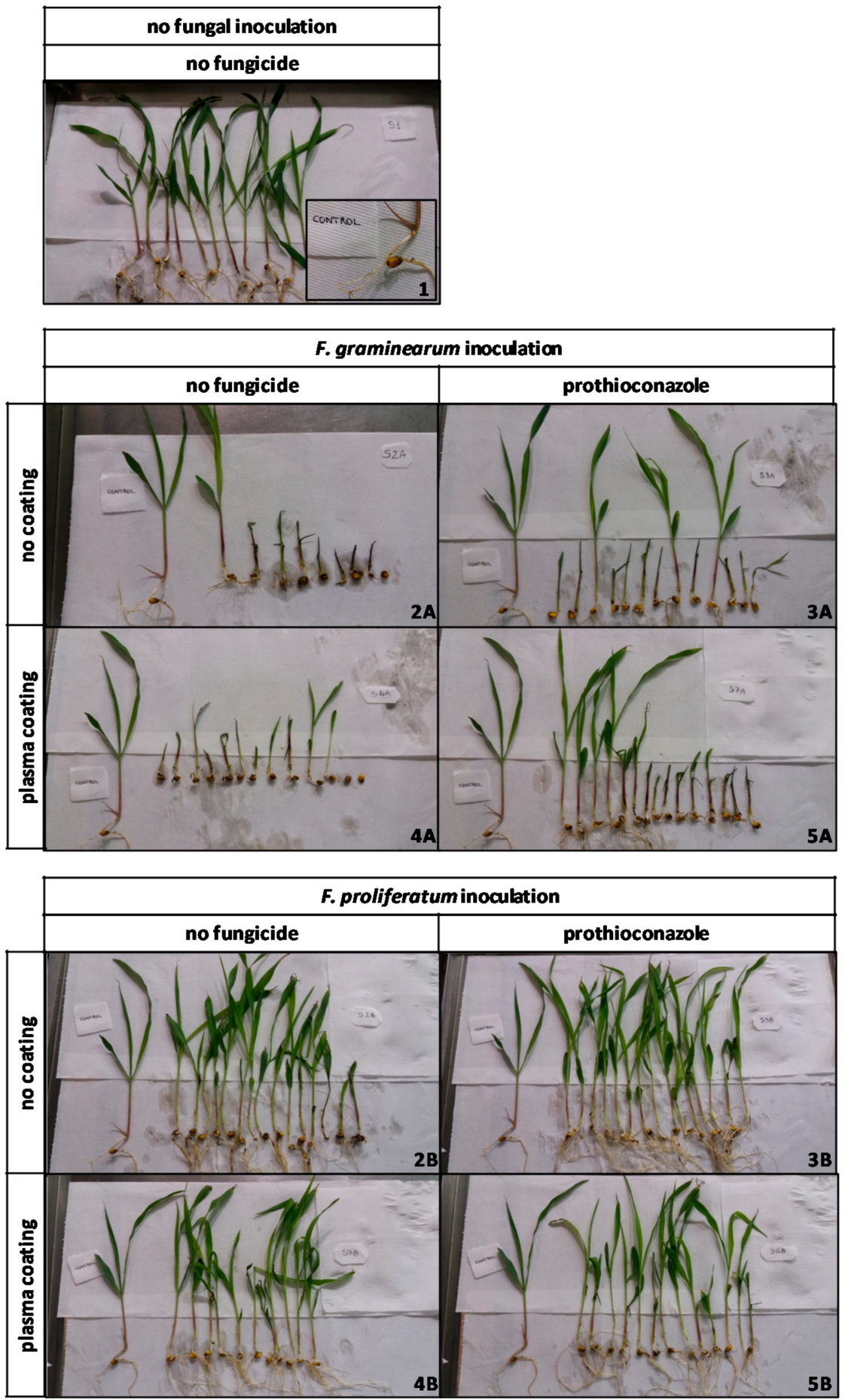

Figure 4. Ten-day-old maize seedlings, unrooted after growing in phytotron. The theses number, representing the different combined treatments, are reported at bottom right of each picture, compared to the control thesis, untreated and not inoculated with Fusarium species.

\section{Discussion}

Fusarium species colonize maize plants through different infection pathways, being infected seeds, crop residues, and soil primary inoculum sources for infection of roots, seedlings, and stems. The data reported in this study provide evidence that $F$. graminearum and F. proliferatum transmission from maize seeds to seedlings in effect occurs. In all theses inoculated with both species, maize stems resulted in being colonized by both fungi. Seed transmission of F. graminearum is well known and widely reported only for 
wheat [36,37], while for maize, only few recent studies exist [38]. Similarly, systemic infection of $F$. proliferatum in maize has been poorly investigated, although a disease cycle similar to F. verticillioides was hypothesized by Munkvold and Desjardins [8]. The Fusarium contamination on maize in the early growth stage leads to seedling decay and reduced crop stands. Moreover, systemic infection during the early growth stage may remain latent, causing disease when suitable conditions occur, and the mycotoxins eventually produced at this stage could translocate from stems to ears and accumulate in the kernels. Therefore, coating of the seed can reduce these risks.

Some authors reported that seed treatments with chemicals can effectively suppress or reduce seed-borne pathogens in maize [39,40], thus reducing crown and stalk rot, while enhancing photosynthesis and improving the vigor of maize plants [41]. However, most data regard F. verticillioides [42]. We reported a high capability of prothioconazole in inhibiting F. verticillioides, F. graminearum, and F. proliferatum in vitro and in field experiments, with respect to other fungicides [33]. Furthermore, prothioconazole encapsulated in the plasma-deposited bilayer has been reported to successfully reduce $F$. graminearum infection in maize seedlings [32]. The current technologies for seed coating provide several advantages, such as the containment of the chemical compounds in confined spaces, and the possibility to reduce the dose of fungicides to control fungal development, with a consequent decreased release of chemicals into the environment. To date, the main application of plasma technology is using cold plasmas at atmospheric pressure deactivating fungal spores and bacteria, therefore improving seed germination and seedling growth to obtain a higher vigor of the plants.

The present innovative work demonstrates the efficacy of embedding prothioconazole sandwiched in a plasma-deposited bilayer coating directly at the surface of the seeds in reducing F. graminearum and F. proliferatum infection of seeds and seedlings. At the same time, we have evaluated the effect on maize seed germination. In the experimental trial in phytotron, after inoculation of $F$. graminearum, a slightly higher seed germination rate was observed in all theses with the plasma-coated maize seeds. On the contrary, plasma-coated seeds inoculated with $F$. proliferatum showed a decreased germination rate.

The plasma coating alone decreased the level of maize stems colonized by F. graminearum from $50 \%$ to $0 \%$, while the colonization by F. proliferatum was reduced to a lower extent, from $62 \%$ to $55 \%$. On the other hand, the stem colonization by F. proliferatum in seeds treated with prothioconazole was not affected by the presence of the plasma coating. On the contrary, F. graminearum growth in stems was found totally inhibited after treatment with prothioconazole alone or encapsulated in a plasma coating. Seed infection of $100 \%$ for both $F$. graminearum and $F$. proliferatum was observed in phytotron trials, except when seeds were treated with prothioconazole alone or encapsulated in a plasma coating. The infection value of seeds treated with prothioconazole decrease to $29 \%$ for F. graminearum and to $85 \%$ for $F$. proliferatum. The efficacy of prothioconazole against $F$. graminearum and $F$. proliferatum was previously proven in the field by Masiello et al. [33]. Our present results obtained with prothioconazole encapsulated in a plasma coating show the reduction of seed infection to $13 \%$ for F. graminearum and to $36 \%$ for F. proliferatum.

Zahoranova et al. [29] reported that the use of cold atmospheric pressure plasma treatments in air on maize seeds allowed a complete devitalization of the native microbiota at the surface of the seeds, showing a consequent potential reduction of the use of hazardous chemicals [29]. Interestingly, a further study has reported the potential of cold plasma processes on roasted coffee to both improve fungal control and induce mycotoxin degradation [28]. However, several studies on the use of cold plasma processes to inactivate toxigenic fungi and detoxify different kinds of seeds (mostly nuts) have shown a dramatic variability of plasma efficacy [43-45], perhaps related to the influence of many parameters such as shape, surface, and size of the seeds, as well as by the sensitivity of each fungal species target. Finally, the homogeneity of the treatments around each single seed clearly plays a role also. 


\section{Materials and Methods}

\subsection{Maize Kernels, Fungicide Application, and Plasma-Deposited Coatings}

Certified uncoated maize seeds of the hybrid variety Marano 0501, marketed by Società Italiana Sementi (SIS Spa, Bologna, Italy), were selected for our experiments.

One hundred randomly selected seeds were tested to evaluate their natural endophytic fungal contamination. Ten seeds per plate, surface sterilized with a $2 \%$ sodium hypochlorite solution and washed twice with sterile distilled water, were placed on potato dextrose agar (PDA) medium. After 5 days of incubation at $25^{\circ} \mathrm{C}$, the fungal colonies originating from infected seeds were counted and morphologically identified. The fungal contamination was expressed as a percentage of contaminated seeds of total tested seeds. The seeds resulted in contamination by F. proliferatum at a low level ( $2 \%)$, while F. graminearum was not detected. Prothioconazole, a systemic fungicide that interferes with fungal sterol biosynthesis, widely used to control Fusarium species on cereal seeds or with foliar applications, was applied at the surface of the seeds as described below.

Part of the maize seeds were treated with the deposition of a plasma-based bilayer coating, as described by Lo Porto et al. [32]. A home-made stainless steel parallel-plate capacitive-coupled RF (13.56 MHz)-driven low pressure plasma reactor was used to deposit a two-layer coating at the surface of maize seeds. Prothioconazole was delivered at the surface of the seeds by spraying $2 \mathrm{~g}$ of a water solution containing $3 \mu \mathrm{L}$ of commercial formulate PROLINE (containing 25\% active ingredient; Bayer Crop Science, Leverkusen, Germany) under shaking conditions, with 30 seeds positioned in a Petri dish. Detail on the reactor and on the deposition processes are published in Lo Porto et al. [32]. The seeds were positioned on the lower, grounded electrode of the reactor, and treated in the following experimental conditions:

Inner coating: $\mathrm{C}_{2} \mathrm{H}_{4}(2.5$ standard $\mathrm{cc} / \mathrm{min}, \mathrm{sccm})$ and $\mathrm{CO}_{2}(5 \mathrm{sccm})$ were used to feed glow discharges of $20 \mathrm{~min}$ duration. The inner coating of about $180 \mathrm{~nm}$ of thickness resulted at the surface of the seeds, characterized by a water contact angle (WCA) of $88 \pm 3^{\circ}$, slightly lower than that of the seeds with $103 \pm 11^{\circ}$. This hydrophilic coating was deposited to enhance the wettability of the seed to the prothioconazole solution.

Outer coating: $\mathrm{C}_{2} \mathrm{H}_{4}(7.5 \mathrm{sccm})$ alone was used to feed glow discharges of $20 \mathrm{~min}$ duration, after the deposition of the inner coating and, for certain seed aliquots, after the administration of the prothioconazole solution. The outer coating of about $800 \mathrm{~nm}$ of thickness resulted onto the seeds, characterized by a WCA of $106 \pm 3^{\circ}$, similar to the hydrophobic pristine value of the untreated seeds.

The seeds treated with the two coatings are often defined as plasma-coated in the whole paper. When applied, prothioconazole is embedded between the two plasmadeposited coatings, by spraying of the above-described solution.

\subsection{Fungal Strains and Preparation of Inoculum}

One strain each for F. graminearum and F. proliferatum was selected from the ITEM Collection of ISPA-CNR (www.ispa.cnr.it/Collection). The F. graminearum ITEM 126 is a highly DON-producing strain [37]; the F. proliferatum strain ITEM 12,072 is a high FBs-producing strain. Both Fusarium strains were used in a previous study to test their sensitivity to different fungicides, including prothioconazole [33]. For each Fusarium strain, a conidial suspension was prepared by scrapping with a sterilized loop the mycelium of 7-day-old colonies dissolved in distilled sterilized water containing $0.01 \%$ of Tween 20 . The conidial suspension was adjusted to $1 \times 10^{5}$ conidia $/ \mathrm{mL}$ for both $F$. graminearum ITEM 126 and $F$. proliferatum ITEM 12072, using the Thoma cell counting chamber. At the same time, five plugs of each 7-day-old colony were added to $200 \mathrm{~g}$ sterilized rice and incubated at $25^{\circ} \mathrm{C}$ for 14 days. This colonized rice was used as inoculum for in planta experimental trial. After incubation, Fusarium colonized rice was gently ground and mixed with sterile river sand in a 1:1 ratio. 


\subsection{In Vitro Experimental Assays}

Two different trials were carried out for $F$. graminearum and F. proliferatum. For each species, four different theses inoculated by the fungal strains were set up and numbers from 2 to 5 were assigned to each thesis, as described in Table 1: thesis with no plasma coating or fungicide treatment; thesis with no plasma coating and fungicide treatment; thesis with plasma coating and no fungicide treatment; thesis with plasma coating and fungicide treatment.

Table 1. Description of theses used for experimental assays in vitro and in planta.

\begin{tabular}{cccc}
\hline Theses & Fungal Inoculation & Seed Coating & Fungicide Application \\
\hline 1 & not inoculated & No & No \\
\hline 2A & & No & No \\
3A & F. graminearum & No & Prothioconazole \\
4A & & Plasma coating & No \\
5A & & Plasma coating & Prothioconazole \\
2B & Fo proliferatum & No & No \\
3B & & Plasma coating & Prothioconazole \\
4B & & Plasma coating & No \\
5B & & &
\end{tabular}

For each thesis, fifteen maize seeds were placed on PDA amended with pentachloronitrobenzene (PCNB). All four theses were inoculated with the conidial suspension: theses inoculated with F. graminearum are denoted by the letter A; theses inoculated with F. proliferatum are denoted by the letter $\mathrm{B}$. The plasma-deposited bilayer coating was utilized on the seeds of Theses 4 and 5; prothioconazole was applied to Theses 3 and 5 (Table 1), as described earlier. All theses were compared with a negative control (Thesis 1), in which seeds were not inoculated with the conidial suspension, and not exposed to any of the plasma and prothioconazole treatments.

In all the inoculated theses, $100 \mu \mathrm{L}$ of conidial suspension was spread on the PDA medium of each Petri dish and 5 maize seeds were then placed in each plate and incubated at $25^{\circ} \mathrm{C}$ under fluorescent light ( $12 \mathrm{~h}$ of photoperiod).

The effect of each treatment in inhibiting the fungal colonization of the seeds was evaluated after 3, 5, 7 and 10 days of incubation. The effects of the treatment on seed germination and on the capability to control maize seedling colonization were also evaluated at the same time. All considered values are expressed in percentages.

To evaluate the systemic colonization of maize seedlings by F. graminearum and $F$. proliferatum, all seedlings were collected after 10 days, surface sterilized with a $2 \%$ sodium hypochlorite solution, washed twice with sterile distilled water, and dried on a sterile filter paper in a laminar flow hood. For each seedling, three small pieces were cut from the seminal root system and from the seedling stem and placed on Petri dishes containing PDA amended with PCNB. Petri dishes were incubated at $25^{\circ} \mathrm{C}$ under fluorescent light $(12 \mathrm{~h}$ of photoperiod). After 6 days of incubation, the contamination level of the two different seedling portions was expressed as a percentage of contaminated tissues on total plated parts.

\subsection{In Planta Experimental Trials in Phytotron}

To test the efficacy of the plasma-based coating embedding the fungicide prothioconazole against $F$. graminearum and $F$. proliferatum in planta, a similar approach was used to that described in Table 1.

For each thesis, 3 pots with max capacity of $2.7 \mathrm{~L}$ (as suggested by the manufacturer) and size of $14 \times 14 \times 16 \mathrm{~cm}^{3}$ were filled with $600 \mathrm{~g}$ of sterilized soil and wet with $250 \mathrm{~mL}$ of sterilized water. Thirty grams of Fusarium-colonized rice mixed with sterile river sand was distributed at the surface of each pot of theses 2, 3, 4 and 5, in a 1:20 ratio of inoculum/soil. 
Thirty grams of sterile river sand without Fusarium-colonized rice was distributed on the control Thesis 1 . The pots were covered with a plastic film and placed in a growth chamber to favor the growth of the fungal inoculum on the soil. After $24 \mathrm{~h}, 15$ maize seeds ( 5 per pot) for each thesis (Table 1) were placed on the surface of the inoculum/sand mixture and covered with $1 \mathrm{~cm}$ of sterile soil. The soil was wet with $150 \mathrm{~mL}$ of sterile water and the pots, covered again with the plastic film, were kept for 4 days in the growth chamber at $23^{\circ} \mathrm{C}$ with a $12 \mathrm{~h}$ light/darkness photoperiod. The pots were then moved to a phytotron, to allow the growth of maize plants in controlled conditions: $23{ }^{\circ} \mathrm{C}, 85 \%$ of relative humidity, 50 lumen, and $12 \mathrm{~h}$ light/darkness photoperiod.

The rate of germination and the general growth of seedlings, in terms of height, vigor, color, and general appearance, were evaluated together with disease symptoms 3, 5, 7 and 10 days after the emergence of the seedlings Seed germination was evaluated by observing the number of seedlings emerged from the total number of seeds sowed, expressed in percentages.

The symptoms of Fusarium infection that were evaluated included the wilting and reddening of the seedlings and the presence of aerial mycelium on stems.

To evaluate the systemic colonization of maize seedlings by F. graminearum and $F$. proliferatum, the seedlings of each thesis were uprooted and collected in paper bags after 10 days. After removing the soil from the roots, the whole seedlings were surface sterilized with a $2 \%$ sodium hypochlorite solution, washed twice with sterile distilled water, and dried on sterile filter paper under a flow hood. From each seedling, small pieces of root and stem were cut of about $5 \mathrm{~mm}$, transferred to Petri dishes containing PDA amended with PCNB, and incubated at $25^{\circ} \mathrm{C}$. The colonization of root seeds and aerial portions of each thesis, expressed as a percentage of colonized tissues on total analyzed portions, was checked after 6 days of incubation.

\subsection{Statistical Analyses}

The SE values for all theses were calculated. Statistical analyses of data were performed using the statistical package STATISTICA v. 6.0 software (StatSoft, Tulsa, OK, USA). A one-way ANOVA was used to test the hypothesis of treatment differentiation with respect to Fusarium of both seminal root systems and stems. The means were then compared using Tukey's honestly significant difference (HSD) test, with a significance level $(p)$ of 0.05 .

\section{Conclusions}

The dramatic reduction of the infected seeds suggests the fungicide coupled with the plasma coating was the most efficient approach for the reduction of contamination and highlights how promising this could be for maize and other seeds. Very likely, the stronger protection effect can be explained by the longer permanence of the fungicide in close contact with the seeds during growth, with the slower release of the molecules in the soil through the bilayer coating. Furthermore, the presence of the coating probably protects the seeds by preventing the infection from the soil and by extending the period of time during which the fungicide is available. Another important achievement of this study is the potential strong reduction of the amount of fungicide used for seed protection. The great attention paid to the environment by the recent Green Deal EU program has clearly defined, as the main object, a significant reduction of chemicals in the next 30 years in the whole EU. Our findings suggest that the application of plasma technology not only offers a potential great improvement for the efficient use of resources in the food industry but would also be a key achievement for improving food safety policy, towards the direction of a more sustainable approach in agriculture.

Author Contributions: Conceptualization, M.M., S.S., C.L.P., F.P., P.F. and A.M.; Data curation, M.M., S.S., C.L.P., F.P., P.F., F.F. and A.M.; Formal analysis, M.M., S.S., C.L.P. and F.P.; Funding acquisition, A.F.L. and A.M.; Investigation, M.M., S.S., C.L.P. and F.P.; Methodology, M.M., S.S., C.L.P., F.P. and P.F.; Project administration, P.F. and A.M.; Resources, M.M., S.S., C.L.P., F.P., P.F., F.F., A.F.L. and A.M.; 
Supervision, P.F. and A.M.; Validation, M.M., S.S., C.L.P. and F.P.; Visualization, M.M., S.S. and A.M.; Writing—original draft, M.M., S.S. and A.M.; Writing—review \& editing, M.M., S.S., F.P., P.F., A.F.L. and A.M. All authors have read and agreed to the published version of the manuscript.

Funding: This research received no external funding.

Institutional Review Board Statement: Not applicable.

Informed Consent Statement: Not applicable.

Data Availability Statement: The data is contained within this article.

Acknowledgments: We thank Giuseppe Cozzi (CNR-ISPA, Bari, Italy) for performing statistical analyses.

Conflicts of Interest: The authors declare no conflict of interest.

\section{References}

1. Edgerton, M.D. Increasing crop productivity to meet global needs for feed, food, and fuel. Plant Physiol. 2009, 149, 7-13. [CrossRef] [PubMed]

2. Orhun, G.E. Maize for Life. Int. J. Food Sci. Nutr. Eng. 2013, 3, 13-16. [CrossRef]

3. Oldenburg, E.; Höppner, F.; Ellner, F.; Weinert, J. Fusarium diseases of maize associated with mycotoxin contamination of agricultural products intended to be used for food and feed. Mycotoxin Res. 2017, 33, 167-182. [CrossRef] [PubMed]

4. Logrieco, A.; Mulè, G.; Moretti, A.; Bottalico, A. Toxigenic Fusarium Species and Mycotoxins Associated with Maize Ear Rot in Europe. Eur. J. Plant Pathol. 2002, 108, 597-609. [CrossRef]

5. Blacutt, A.A.; Gold, S.E.; Voss, K.A.; Gao, M.; Glenn, A.E. Fusarium verticillioides: Advancements in understanding the toxicity, virulence, and niche adaptations of a model mycotoxigenic pathogen of maize. Phytopathology 2018, 108, 312-326. [CrossRef] [PubMed]

6. Gai, X.; Dong, H.; Wang, S.; Liu, B.; Zhang, Z.; Li, X.; Gao, Z. Infection cycle of maize stalk rot and ear rot caused by Fusarium verticillioides. PLoS ONE 2018, 13, e0201588. [CrossRef] [PubMed]

7. Munkvold, G.P.; Carlton, W.M. Influence of inoculation method on systemic Fusarium moniliforme infection of maize plants grown from infected seeds. Plant Dis. 1997, 81, 211-216. [CrossRef]

8. Munkvold, G.P.; Desjardins, A.E. Fumonisins in maize: Can we reduce their occurrence? Plant Dis. 1997, 81, 556-565. [CrossRef]

9. Fallahi, M.; Saremi, H.; Javan-Nikkhah, M.; Somma, S.; Haidukowski, M.; Logrieco, A.F.; Moretti, A. Isolation, Molecular Identification and Mycotoxin Profile of Fusarium Species Isolated from Maize Kernels in Iran. Toxins 2019, 11, 297. [CrossRef]

10. Zhou, D.; Wang, X.M.; Chen, G.K.; Sun, S.; Yang, Y.; Zhu, Z.D.; Duan, C.X. The major Fusarium species causing maize ear and kernel rot and their toxigenicity in Chongqing, China. Toxins 2018, 10, 90. [CrossRef]

11. Marín, S.; Magan, N.; Ramos, A.J.; Sanchis, V. Fumonisin-Producing Strains of Fusarium: A Review of Their Ecophysiology. J. Food Prot. 2004, 67, 1792-1805. [CrossRef] [PubMed]

12. Logrieco, A.; Moretti, A.; Ritieni, A.; Bottalico, A.; Corda, P. Occurrence and toxigenicity of Fusarium proliferatum from preharvest maize ear rot, and associated mycotoxins in Italy. Plant Dis. 1995, 79, 727-731. [CrossRef]

13. Proctor, R.H.; Desjardins, A.E.; Moretti, A. Biological and chemical complexity of Fusarium proliferatum. In The Role of Plant Pathology in Food Safety and Food Security; Springer: Dordrecht, The Netherlands, 2009; pp. 97-111.

14. Marasas, W.F.O.; Thiel, P.G.; Rabie, C.J.; Nelson, P.E.; Toussoun, T.A. Moniliformin production in Fusarium section Liseola. Mycologia 1986, 78, 242-247. [CrossRef]

15. Moretti, A.; Logrieco, A.; Bottalico, A.; Ritieni, A.; Randazzo, G. Production of beauvericin by Fusarium proliferatum from maize in Italy. Mycotoxin Res. 1994, 10, 73-78. [CrossRef] [PubMed]

16. Ritieni, A.; Fogliano, V.; Randazzo, G.; Scarallo, A.; Logrieco, A.; Moretti, A.; Mannina, L.; Bottalico, A. Isolation and characterization of fusaproliferin, a new toxic metabolite from Fusarium proliferatum. Nat. Toxins 1995, 3, 17-20. [CrossRef]

17. Miller, J.D.; Savard, M.E.; Schaafsma, A.W.; Seifert, K.A.; Reid, L.M. Mycotoxin production by Fusarium moniliforme and Fusarium proliferatum from Ontario and occurrence of fumonisin in the 1993 corn crop. Can. J. Plant Pathol. 1995, 17, $233-239$.

18. Pfordt, A.; Romero, L.R.; Schiwek, S.; Karlovsky, P.; von Tiedemann, A. Impact of Environmental Conditions and Agronomic Practices on the Prevalence of Fusarium Species Associated with Ear- and Stalk Rot in Maize. Pathogens 2020, 9, 236. [CrossRef]

19. Murillo-Williams, A.; Munkvold, G.P. Systemic infection by Fusarium verticillioides in maize plants grown under three temperature regimes. Plant Dis. 2008, 92, 1695-1700. [CrossRef]

20. Weltmann, K.D.; Kolb, J.F.; Holub, M.; Uhrlandt, D.; Simek, M.; Ostrikov, K.; Hamaguchi, S.; Cvelbar, U.; Cernak, M.; Locke, B.; et al. The future in Plasma Science and Technology. Plasma Process. Polym. 2019, 16, e1800118. [CrossRef]

21. Bekeschus, S.; Favia, P.; Robert, E.; von Woedtke, T. White paper on plasma for medicine and hygiene: Future in plasma health sciences. Plasma Process. Polym. 2019, 16, e1800033. [CrossRef]

22. Trizio, I.; Trulli, M.G.; Lo Porto, C.; Pignatelli, D.; Camporeale, G.; Palumbo, F.; Sardella, E.; Gristina, R.; Favia, P. Plasma Processes for Life Sciences. In Elsevier Reference Module in Chemistry, Molecular Sciences and Chemical Engineering; Reedijk, J., Ed.; Elsevier: Waltham, MA, USA, 2018. [CrossRef] 
23. Khatami, S.; Ahmadinia, A. Increased germination and growth rates of pea and Zucchini seed by FSG plasma. J. Theor. Appl. Phys. 2018, 12, 33-38. [CrossRef]

24. Kumar, R.; Singh, P.; Kumar, S. Plasma Treatment-A Tool to Improve Seed Quality-A Review. Adv. Res. 2017, 12, 1-6. [CrossRef]

25. Sivachandiran, L.; Khacef, A. Enhanced seed germination and plant growth by atmospheric pressure cold air plasma: Combined effect of seed and water treatment. RSC Adv. 2017, 7, 1822-1832. [CrossRef]

26. Brasoveanu, M.; Nemtanu, M.R.; Surdu-Bob, C.; Karaca, G.; Erper, I. Effect of Glow Discharge Plasma on Germination and Fungal Load of Some Cereal Seeds. Rom. Rep. Phys. 2015, 67, 617-624.

27. Filatova, I.; Azharonok, V.; Lushkevich, V.; Zhukovsky, A.; Spasić, K.; Živković, S.; Puač, N.; Lazović, S.; Malović, G.; Petrović, Z. Plasma seeds treatment as a promising technique for seed germination improvement. In Proceedings of the 31st ICPIG, Granada, Spain, 14-19 July 2013; Available online: http:/ / icpig2013.net/papers/127_2.pdf (accessed on 9 June 2021).

28. Casas-Junco, P.P.; Solís-Pacheco, J.R.; Ragazzo-Sánchez, J.A.; Aguilar-Uscanga, B.R.; Bautista-Rosales, P.U.; Calderón-Santoyo, M. Cold Plasma Treatment as an Alternative for Ochratoxin a Detoxification and Inhibition of Mycotoxigenic Fungi in Roasted Coffee. Toxins 2019, 11, 337. [CrossRef]

29. Zahoranova, A.; Hoppanova, L.; Simoncicova, J.; Tuekova, Z.; Medvecka, V.; Hudecova, D.; Kaliňáková, B.; Kováčik, D.; Černák, M. Effect of cold atmospheric pressure plasma on maize seeds: Enhancement of seedlings growth and surface microorganisms inactivation. Plasma Chem. Plasma Process. 2018, 38, 969-988. [CrossRef]

30. Jo, Y.K.; Cho, J.; Tsai, T.C.; Staack, D.; Kang, M.H.; Roh, J.H.; Shin, D.B.; Cromwell, W.; Gross, D. A non-thermal plasma seed treatment method for management of a seedborne fungal pathogen on rice seed. Crop Sci. 2014, 54, 796-803. [CrossRef]

31. Puač, N.; Gherardi, M.; Shiratani, M. Plasma agriculture: A rapidly emerging field. Plasma Process. Polym. 2018, 15, e1700174. [CrossRef]

32. Lo Porto, C.; Palumbo, F.; Somma, S.; Masiello, M.; Moretti, A.; Fracassi, F.; Favia, P. Plasma-assisted deposition of fungicide containing coatings for encapsulation and protection of maize seeds. Plasma Process. Polym. 2019, 16, e1900022. [CrossRef]

33. Masiello, M.; Somma, S.; Ghionna, V.; Logrieco, A.F.; Moretti, A. In Vitro and in Field Response of Different Fungicides against Aspergillus flavus and Fusarium Species Causing Ear Rot Disease of Maize. Toxins 2019, 11, 11. [CrossRef]

34. Da Ponte, G.; Sardella, E.; Fanelli, F.; Van Hoeck, A.; d'Agostino, R.; Paulussen, S.; Favia, P. Atmospheric Plasma Deposition of different organic films for biomedical applications. Surf. Coat. Technol. 2011, 205, S525. [CrossRef]

35. Da Ponte, G.; Sardella, E.; Fanelli, F.; d'Agostino, R.; Favia, P. Trends in surface engineering of biomaterials: Atmospheric pressure plasma deposition of coatings for biomedical applications. Eur. Phys. J.-Appl. Physics 2011, 56, 24023. [CrossRef]

36. Duthie, J.A.; Hall, R. Transmission of Fusarium graminearum from seeds to stems of winter wheat. Plant Pathol. 1987, $36,3337$. [CrossRef]

37. Moretti, A.; Panzarini, G.; Somma, S.; Campagna, C.; Ravaglia, S.; Logrieco, A.F.; Solfrizzo, M. Systemic growth of F. graminearum in wheat plants and related accumulation of deoxynivalenol. Toxins 2014, 6, 1308-1324. [CrossRef] [PubMed]

38. Kabeere, F.; Hampton, J.G.; Hill, M.J. Transmission of Fusarium graminearum (Schwabe) from maize seeds to seedlings. Seed Sci. Technol. 1997, 25, 245-252.

39. Burlakoti, R.R.; Burlakoti, P. Management of maize ear rot complex caused by Fusarium species in field using host resistance and seeds treatment chemicals. J. Crop Prot. 2015, 4, 453-462.

40. Munkvold, G.P.; O'Mara, J.K. Laboratory and growth chamber evaluation of fungicidal seed treatments for maize seedling blight caused by Fusarium species. Plant Dis. 2002, 86, 143-150. [CrossRef]

41. Rodriguez-Brljevich, C.; Kanobe, C.; Shanahan, J.F.; Robertson, A.E. Seed treatments enhance photosynthesis in maize seedlings by reducing infection with Fusarium spp. and consequent disease development in maize. Eur. J. Plant Pathol. 2010, 126, 343-347. [CrossRef]

42. Galperin, M.; Graf, S.; Kenigsbuch, D. Seeds treatment prevents vertical transmission of Fusarium moniliforme, making a significant contribution to disease control. Phytoparasitica 2003, 31, 344-352. [CrossRef]

43. Siciliano, I.; Spadaro, D.; Prelle, A.; Vallauri, D.; Cavallero, M.C.; Garibaldi, A.; Gullino, M.L. Use of cold atmospheric plasma to detoxify hazelnuts from aflatoxins. Toxins 2016, 8, 125. [CrossRef]

44. Ouf, S.A.; Mohamed, A.A.H.; El-Sayed, W.S. Fungal Decontamination of Fleshy Fruit Water Washes by Double Atmospheric Pressure Cold Plasma. CLEAN-Soil Air Water 2015, 44, 134-142. [CrossRef]

45. Basaran, P.; Basaran-Akgul, N.; Oksuz, L. Elimination of Aspergillus parasiticus from nut surface with low pressure cold plasma (LPCP) treatment. Food Microbiol. 2008, 25, 626-632. [CrossRef] [PubMed] 